\title{
Psicoanálisis, Psicoterapia psicoanalítica y Estigma
}

\author{
Adrián Ortíz ${ }^{1}$ y Myrthala Juárez Treviño ${ }^{2}$ \\ CENTRO DE EDUCACION EINVESTIGACION EN PSICOANALISIS (CEIP), MONTERREY, MEXICO
}

En México es muy difícil que las personas acudan a psicoterapia o psicoanálisis, porque existe un miedo de las personas hacia los psicoterapeutas y psicoanalistas. Existe un prejuicio sobre la enfermedad mental. Creemos que la intersubjetividad puede ser un puente entre la sociedad civil y los profesionales del psicoanálisis.

\section{Palabras clave: Psicoanálisis, Estigma}

In Mexico is too hard that people take psychotherapy or psychoanalysis, because there exist a scaring, people afraid from psychotherapist and psychoanalist. There exist a pre jugment, an idea about mental disease. We think that intersubjetivity may be a brige between the social civil and the professionals in psychoanalysis.

Key Words: Psychoanalysis, Stigma

English Title: Psychoanalysis, Psychoanalytic psychotherapy and Stigma

\section{Cita bibliográfica / Reference citation:}

Ortíz, A. y Juárez Treviño, M. (2019). Psicoanálisis, Psicoterapia psicoanalítica y Estigma. Clínica e Investigación Relacional, 13 (2): 510-514. [ISSN 1988-2939] [Recuperado de www.ceir.info ] DOI:

10.21110/19882939.2019.130212

\footnotetext{
${ }^{1}$ Médico-psiquiatra por la Universidad de Monterrey (UDEM), candidato en entrenamiento en psicoanálisis por el Centro de Educación e Investigación en Psicoanálisis (CEIP). Actualmente Secretario del Colegio de Psiquiatría y Psicofarmacología de Nuevo León A.C.

${ }^{2}$ Doctora en Medicina. Médico Cirujano Partero Universidad Autónoma de Nuevo León (UANL), especialidad en Psiquiatría y Psiquiatría Infantil y de la Adolescencia. UANL. Psicoanalista. Miembro de la International Psychoanalytic Association, la Asociación Psiquiátrica Mexicana (APM) yla Asociación Mexicana de Psiquiatría Infantil (AMPI)
} 


\section{Sobre el estigma en psicoanálisis y la psicoterapia.}

Este artículo va dirigido a combatir el estigma que la sociedad mexicana tiene hacia la psicoterapia y hacia el psicoanálisis. Ya que son herramientas poderosas para tratar las enfermedades mentales, y específicamente los trastornos de personalidad. Entidades nosológicas que impactan de manera gravísima tanto para la salud y economía personal como en la sociedad en general.

Las estadísticas no mienten, la mayoría de las personas que cursan con un cuadro de depresión mayor no se atienden adecuadamente con un profesional en salud mental, las tasas de suicidio se incrementan, afectando a las personas más jóvenes. La idea que se tiene sobre la psicoterapia no pasa de ser una caricatura de las películas de Hollywood, como por ejemplo la que hizo Woody Allen.

En algunas ocasiones se le puede considerar al psicoanálisis hasta falto de cientificidad. Y en otras como una última salvación para enfermedades tan desgastantes como los son los trastornos de la personalidad.

Considero que el psicoanálisis y la psicoterapia, como métodos de tratamiento enfocados a ayudar al sufrir de las personas tienen una aplicación muy práctica y que tienen gran impacto tanto para la vida personal de los pacientes como en su entorno (familia, amigos, trabajo, sociedad).

En el XXIX congreso de la Asociación Psiquiátrica de América Latina ( $A P A L)$, realizado en Guatemala (2018) se enfocó el tema principal sobre el estigma en salud mental, el estigma en el tratamiento en los trastornos de personalidad, y el estigma sobre los tratamientos en psiquiatría y psicoterapia. En donde se abordó como puntos principales el estigma público y el autestigma. El estigma publico sería el que comprende todo prejuicio y discriminación adoptada por la sociedad y dirigida contra las personas que padecen una enfermedad mental. Y el autoestigma el cual se refiere al daño que sucede cuando las personas que sufren una enfermedad mental interiorizan los prejuicios sociales existentes contra ellos.

La educación sobre las principales enfermedades mentales es muy escasa en lo que se refiere a la propaganda que se le hace en medios de comunicación masiva, y que actualmente existe mucha mala información sobre cómo se deben de atender adecuadamente un problema en salud mental, no se diga un trastorno de personalidad, o tan solo ciertas dificultades de la vida cotidiana que amerita una orientación por parte del psicoterapeuta.

Lo que el psicoanálisis le ha aportado a la psicoterapia desde sus inicios ha sido definitivo para la comprensión de la psique humana. Desde la concepción de un inconsciente y que 
todas las personas tienen un inconsciente y que es éste en donde residen los conflictos intrapsíquicos que enferman a las personas. Ya el mismo Freud a comienzos del siglo $X X$, nos enseñó que la mayoría de las personas no se daban cuenta del origen de sus síntomas, y que es precisamente por medio del psicoanálisis que se puede llegar hasta la raíz de los problemas, siendo un camino tortuoso que contiene varios obstáculos.

En el trabajo de los psicoterapeutas existe también un compromiso social. Yo creo que cada vez más se está abandonando esa idea que se tiene sobre el psicoterapeuta, que se encuentra hundido en su consultorio esperando a que lleguen sus pacientes, pero que no existe un contacto entre él y la sociedad, que no va a espacios públicos, a dar platicas psicoeducativas, que "no sale al campo a ensuciarse las botas", es una idea que se está abandonando cada vez, por lo menos en nuestra sociedad mexicana. Hablo de mi experiencia personal y de varios de mis colegas psiquiatras y psicoterapeutas, que tratamos de buscar espacios públicos para psicoeducar a la población.

Creo que como profesionistas y como personas públicas que somos tenemos la responsabilidad enorme de preocuparnos seriamente por educar a nuestra comunidad, el hablar sobre psicoterapia, en que consiste, el hablar sobre el psicoanálisis y los beneficios que produce en las personas, el hablar sobre el estigma en la psicoterapia y tratar de derrumbar diversos tabúes.

\section{Enfoque intersubjetivo como apoyo para derribar el estigma.}

Los trastornos de personalidad y el malestar ciudadano han sido temas de particular interés y que han sido piezas clave para mi inclinación hacia el psicoanálisis.

El psicoanálisis, en una de sus corrientes más contemporáneas como lo es el enfoque intersubjetivo y el psicoanálisis relacional, es un modelo que creo que puede ayudar a eliminar la imagen peyorativa que se tiene sobre el psicoanálisis y la psicoterapia en la sociedad.

Siento que tanto la psicoterapia como el psicoanálisis muchas veces son considerados como métodos obsoletos o de épocas pasadas, que se alejan del ámbito médico. Ya que en nuestros tiempos existe un cuerpo muy pesado de especialistas que tienden a manejar meramente con un tratamiento farmacológico a los diversos trastornos psiquiátricos. En los cuales el psicoanálisis y la psicoterapia pueden ayudar e incluso curar. 
El psicoanálisis como cuerpo teórico puede aportar mucho desde la interpretación de fenómenos sociales, análisis de la cultura occidental moderna, tan solo por citar algunos ejemplos.

Todos como personas sociales que somos, "nadie puede ser una isla", tenemos una amplia gama de relaciones, tenemos una matriz relacional. Nos relacionamos con nuestros seres queridos, nos relacionamos con nuestros amigos, nos relacionamos con nuestros compañeros de trabajo, y la gama de relaciones no tiene fin. En esta trama de relaciones se desenvuelve nuestra persona. Nuestra forma de sentir y de pensar se desenvuelve en el desarrollo de todas estas relaciones. Nuestra experiencia subjetiva de nuestra persona tiene impacto con los demás y con nosotros mismos. Por ello creo que específicamente el marco teórico del psicoanálisis relacional tiene mucho que enseñarnos como personas pensantes que somos, y asi poder entender los diversos trastornos de personalidad que se padecen.

Creo que desde el marco teórico referencial de la intersubjetividad en psicoanálisis nos puede ayudar mucho a tratar de comprender esta reacción de la población hacia la psicoterapia y hacia el psicoanálisis. Ya que en muchos escenarios o en opiniones públicas se tiene a imaginar al terapeuta o psicoanalista como distante, interpretando las fantasías, sueños, síntomas del paciente, como si se tratase de un ambiente de laboratorio en donde el laboratorista (terapeuta - analista), observa los fenómenos en los pacientes. Y creo que esa imagen tiene mucha fuerza en el juicio y opinión de la población general acerca de lo que un terapeuta o un analista hacen.

Entonces, si nos guiamos de la mano de la teoría de la intersubjetividad, que parte del hecho de que no se trata meramente de un analista observador, completamente neutral y anónimo atendiendo a un paciente, sino más bien de una persona con preparación completamente profesional en psicoanálisis y que puede hacer uso de sus afectos y conocimientos en mejora de la salud mental de otra persona, igual que él. Si pudiera llegar un poco más de esta "nueva imagen" del trabajo activo del analista - terapeuta, hacia la población general, creo que sería un gran avance y tendría un mayor acercamiento, más real de lo que en verdad puede ayudar la psicoterapia y el psicoanálisis a nuestros pacientes y a toda nuestra población mexicana.

Estoy hablando de que esta nueva imagen se debe de crear primeramente en las enseñanzas de las nuevas generaciones de analistas jóvenes en México, en donde se considere tanto en las residencias de la especialidad en psiquiatría dentro de las materias impartidas, así como también a las nuevas generaciones de psicoanalistas mexicanos. 
Es importante primeramente entender cuál es la visión de la teoría intersubjetiva, desde sus inicios. El marco de referencia que nos puede aportar grandes conocimientos para el entendimiento del sufrimiento humano. El enfoque intersubjetivo parte del reconocimiento del otro, la identificación del otro, que al igual que la misma persona del analista tiene afectos, conocimientos y maneras de ser y de pensar. "Solo lo humano puede ayudar a lo humano".

Para ello es menester retomar los trabajos de Mitchell, Stern, Andre Green, solo por citar algunos.

Joan Coderch, psicoanalista español, contemporáneo, menciona que el psicoanálisis relacional ayuda al paciente a vivir de una manera "más viva su vida". Ya que el marco teórico del psicoanálisis relacional parte del hecho de que el paciente desde su nacimiento es un ser biológicamente social, y que desde el nacimiento tiene interacciones reciprocas tanto con su medio ambiente como con los padres, y que son estas interacciones lo que va a determinar la experiencia subjetiva de su vida.

Por ello, continua Coderch, esta referencia teórica no ayudara a comprender y a saber por qué el paciente responde de tal o cual manera a cualquier situación. El psicoanálisis relacional dirige su acción al logro de cambios en el inconsciente que se encuentra constituido por el enorme caudal de contenido (experiencias interpersonales, aprendizajes, hábitos, esquemas mentales, conocimientos diversos, etc).

Descartar los contenidos ocultos de las experiencias subjetivas y considerar únicamente lo visible y lo meramente superficial de las vidas sería el error más grave que podríamos hacer.

\section{REFERENCIAS}

Coderch, J. (2016). Teoría y técnica de la psicoterapia psicoanalítica. Herder Editorial. 2016. España.

Juarez-Treviño, M. (2019). Does psychoterapy change the brain functioning?". American Journal of Biomedical Science and Research. 2019.

Mascayano F, Lips Castro,W, et - al. (2015). Estigma hacia los trastornos mentales: características e intervenciones. Salud Mental vol.38 no.1 México. 2015.

VV.AA. (2004). La salud mental en México, Servicio de Investigación y Análisis. División de política social. Camara de Diputados. LIX Legislatura. 2004

Original recibido con fecha: 18/6/2019 Revisado: 29/10/2019 Aceptado: 30/10/2019 